\title{
Complex X-Ray Spectral Variability in Mini-BAL QSOs
}

\author{
Margherita Giustini ${ }^{1,2,3}$, Massimo Cappi $^{3}$, George Chartas $^{4,1}$, \\ Michael Eracleous ${ }^{1}$, Giorgio G. C. Palumbo ${ }^{2}$, and Cristian Vignali ${ }^{2}$ \\ ${ }^{1}$ Department of Astronomy \& Astrophysics, Pennsylvania State University, \\ University Park, PA 16802, USA \\ ${ }^{2}$ Dipartimento di Astronomia, Università di Bologna, via Ranzani 1, I-40127 Bologna, Italy \\ ${ }^{3} \mathrm{INAF} /$ Istituto di Astrofisica Spaziale e Fisica cosmica, \\ via Gobetti 101, I-40138 Bologna, Italy \\ ${ }^{4}$ Department of Physics and Astronomy, College of Charleston, Charleston, SC 29424, USA
}

\begin{abstract}
Multiple XMM-Newton observations of the mini-BAL QSOs PG $1351+640$ and PG 1126-041 revealed dramatic and complex spectral variability on different timescales, providing clues about the physical mechanism responsible for outflows in quasars.
\end{abstract}

Keywords. galaxies: active, X-rays: individual (PG 1351+640, PG 1126-041)

Quasar outflows provide a feedback mechanism between supermassive black holes and their environments. We performed temporally resolved X-ray spectral analysis on two mini-BAL QSOs, PG 1351+640 $(z \sim 0.09)$ and PG 1126-041 $(z \sim 0.06)$; these sources host ultraviolet absorbing gas outflowing at $v \sim 3500 \mathrm{~km} \mathrm{~s}^{-1}$ and $v \sim 5000 \mathrm{~km} \mathrm{~s}^{-1}$, respectively. The investigation of their X-ray properties provides insights into the physical mechanism responsible for launching and accelerating such outflows.

The main results of multiple $X M M-$ Newton observations are the following:

- High column densities ( $\left.\sim 10^{23} \mathrm{~cm}^{-2}\right)$ of $X$-ray absorbing gas are detected. Different phases of gas are detected in each source, with ionization parameters spanning the range $\sim 10-100 \mathrm{erg} \mathrm{cm} \mathrm{s}^{-1}$. This gas is only partially covering the continuum source.

- Strong X-ray spectral variability on different timescales (years, months, and hours) is detected. This variability can be well reproduced by variations of physical parameters (column density, covering factor) of the ionized absorbers.

- The optical-to-X-ray spectral index is found to be significantly variable. The $\alpha_{\text {ox }}$ parameter, measured simultaneously for each epoch thanks to the Optical Monitor aboard $X M M-N e w t o n$, is variable. What drives the changes in $\alpha_{\mathrm{ox}}$ is the strong X-ray variability.

- The detection of a high velocity X-ray outflow $\left(v \sim 15000 \mathrm{~km} \mathrm{~s}^{-1}\right)$ in PG 1126-041 is made possible thanks to a very high signal-to-noise-ratio observation.

Overall, these observational results are in qualitative agreement with radiatively driven accretion disk wind models. Further insights into the physics of quasar outflows would be provided by checking whether such models can also quantitatively reproduce the observed complexities.

\section{Acknowledgements}

We acknowledge financial support from NASA grant NNX08AB71G and ASI contract I/088/06/0. MG acknowledge the AAS/NSF Travel Grant. ME acknowledges support from NSF grant AST-0807993. 